\title{
A Large-Eddy Simulation Study of Anisotropy in Fair-Weather Cumulus Cloud Fields
}

\author{
LAURA M. HiNKELMAN* \\ Department of Meteorology, The Pennsylvania State University, University Park, Pennsylvania \\ BJORN STEVENS \\ Department of Atmospheric Sciences, University of California, Los Angeles, Los Angeles, California \\ K. FrankLin Evans \\ Program in Atmospheric and Oceanic Sciences, University of Colorado, Boulder, Colorado
}

(Manuscript received 12 March 2004, in final form 9 November 2004)

\begin{abstract}
Causes of anisotropy in fair-weather cumulus cloud fields were investigated using quantitative measures of anisotropy and a large-eddy simulation (LES) model. Case six of the Global Energy and Water Cycle Experiment (GEWEX) Cloud System Study Working Group 1 was used as the standard model scenario. This case represents radiatively forced development of cumulus clouds over the southern Great Plains. Cloud formation under a variety of environmental conditions was simulated and the degree of anisotropy in the output fields was calculated as a function of spatial scale. Wind shear was found to be the single greatest factor in the development of both vertically tilted and horizontally stretched cloud structures. Other factors included mean wind speed, initial water vapor mixing ratio, and the magnitude of the surface forcing.
\end{abstract}

\section{Introduction}

Accurate computation of radiative transfer through clouds is important to understanding and predicting the earth's climate (Houghton et al. 2001). Historically, clouds have been modeled as homogeneous slabs with planar boundaries parallel to the earth's surface, but this approximation is known to have limited application (Harshvardhan and Thomas 1984; Welch and Wielicki 1984). Ignoring the structure and variability of cloud fields can lead to biases in radiative transfer calculations. This is especially likely for cumulus or other broken cloud systems, which most clearly deviate from the plane-parallel assumptions.

The effects of a variety of cloud features, such as horizontal variations of optical depth in stratus or stratocumulus cloud decks (Cahalan et al. 1994; Marshak et al. 1995; Zuidema and Evans 1998), cloud field broken-

\footnotetext{
* Current affiliation: National Institute of Aerospace, Hampton, Virginia.
}

Corresponding author address: Dr. Laura Hinkelman, NASA Langley Research Center, Mail Stop 420, Hampton, VA 236812199.

E-mail: 1.m.hinkelman@larc.nasa.gov ness and cumulus cloud geometry (Busygin et al. 1973; McKee and Cox 1974; Aida 1977; Harshvardhan and Thomas 1984; Welch and Wielicki 1984), and cloud-top height fluctuations (Várnai and Davies 1999), on solar radiative transport have been investigated. One feature that has received little attention is the degree of orientation, whether in the horizontal or vertical plane, present in cloud field structures. Since even the casual observer frequently notices preferred directions (or anisotropy) in cloud fields, consideration of the radiative impact of such structure seems advisable. Barker and Davies (1992) and Barker (1994) have briefly discussed the interaction of solar radiation and tilted or stretched cloud structures. A more systematic study of these effects is now under way in our group and will be described in a subsequent paper.

For these radiative transfer studies to be meaningful, however, they must be based on the natural range of anisotropy in broken cloud fields. Knowledge of the conditions under which cloud field anisotropy occurs and the prevalence of these conditions is also needed to assess the climatological significance of anisotropy's radiative effects. However, systematic investigation of the range and causes of anisotropy in cloud fields at the scale of interactions between clouds and solar radiation (tens to hundreds of meters) is lacking, in part because 
this is also the limit of resolution of numerical cloud models and remote sensing instruments.

Many papers have been written about the causes of organization in cumulus or stratocumulus cloud fields (e.g., Kuettner 1971; LeMone 1973; Grossman 1982; Shirer 1986; Christian 1987; Sykes and Henn 1989; Weckwerth et al. 1997; Glendening 2000), but most of these are concerned with forms of organization occurring on the mesoscale, such as cloud streets. The smaller scale structure of individual cumulus clouds and sparse cumulus fields has received less attention. Research in this area has focused on flux budget profiles in single clouds (Heymsfield et al. 1978; Brown 1999) or the number, size, and distribution of clouds in a scene (Cho 1978; Joseph 1985; Sengupta et al. 1990; Zhu et al. 1992). Good discussions of stretching and tilting of clouds of suspended particles are found in the dispersion literature (Saffman 1962; Taylor 1982; Mason 1992) going back to the 1960s. A drawback of these works is that there is no quantification of anisotropy or systematic correlation with environmental features, even when stretching and tilting of scalar clouds are explicitly discussed (Taylor 1982). In addition, water is not truly a passive scalar during cloud formation because it condenses and is subject to evaporation during this process.

Although they do not specifically address the causes of anisotropy in cumulus cloud fields, many of these articles suggest sources of organization and deformation that may be relevant here. Significant winds have been cited as contributing to roll convection (Christian 1987) and might be expected to stretch clouds in one direction. Speed shear and buoyancy are considered in nearly all attempts to explain the formation of roll clouds (LeMone 1973; Grossman 1982; Shirer 1986; Sykes and Henn 1989; Weckwerth et al. 1997; Glendening 2000) and should be considered here as well-the changing of wind speed with height could cause tilt by displacing cloud layers horizontally while vertical momentum from buoyant forces could counteract the effects of shear. Speed and directional shear are also discussed in the dispersion literature as sources of particle cloud deformation (Saffman 1962; Taylor 1982; Mason 1992).

This paper describes a study of the causes of anisotropy in fair-weather cumulus cloud fields at scales of tens to hundreds of meters. Numerical modeling is employed because it allows the conditions of cloud formation to be closely controlled. Once a basic scenario has been established, the environmental conditions are systematically varied, with an emphasis on those factors from the literature described above. New measures of anisotropy are applied to the simulated cloud fields, allowing quantitative assessment of the resulting anisotropy and improved evaluation of the correlation between cloud field geometry and the ambient conditions during cloud formation.

\section{Anisotropy measures}

Isotropy is defined as the lack of a preferred direction in the structure of a field. However, gravity clearly establishes a preferred direction in the atmosphere. For this reason, we define two specific and orthogonal types of anisotropy for use in describing cloud fields. The first is the degree to which structures in a horizontal plane are elongated, which we term horizontal anisotropy. The second is the extent to which cloud structure is skewed or offset horizontally as a function of height. This we term tilt. Separate measures are used to quantify these two types of anisotropy.

\section{a. Horizontal anisotropy}

To quantify the degree to which a two-dimensional field depends on direction, we define the anisotropy parameter (AP). This parameter consists of an amplitude, which indicates the strength of the directionality, and a direction, which indicates the predominant orientation of structures in the field. It is calculated as a function of spatial scale and evaluated using a statistical significance test.

The method used to calculate the anisotropy parameter for a given two-dimensional array, here a liquid water path field, is illustrated in Fig. 1. The power spectrum $S(f, \phi)$, where $f$ is spatial frequency and $\phi$ is azimuthal angle, of the field is first obtained by taking the square of the amplitude of the two-dimensional fast Fourier transform (FFT) of the field. The power spectrum is next divided into half-octave spatial frequency bands. The power in each band is then decomposed into its vertical/horizontal (north-south/east-west) and diagonal (NW-SE/SW-NE) components according to the integrals

$$
M_{c, n}=\int_{f_{n-1}}^{f_{n}} \int_{0}^{2 \pi} S(f, \phi) \cos (2 \phi) d \phi d f
$$

and

$$
M_{s, n}=\int_{f_{n-1}}^{f_{n}} \int_{0}^{2 \pi} S(f, \phi) \sin (2 \phi) d \phi d f,
$$

where the subscripts $s$ and $c$, respectively, denote the sine and cosine weighted terms and $f_{n}$ is the outer boundary of spatial frequency band $n$. If the original field is perfectly isotropic, its power spectrum will be axially symmetric and these integrals will each yield zero. Otherwise, $M_{c, n}$ and $M_{s, n}$, when normalized by the total power

$$
M_{0, n}=\int_{f_{n-1}}^{f_{n}} \int_{0}^{2 \pi} S(f, \phi) d \phi d f
$$

in each band, indicate the fraction of the power in that band that is aligned along or perpendicular to the hori- 

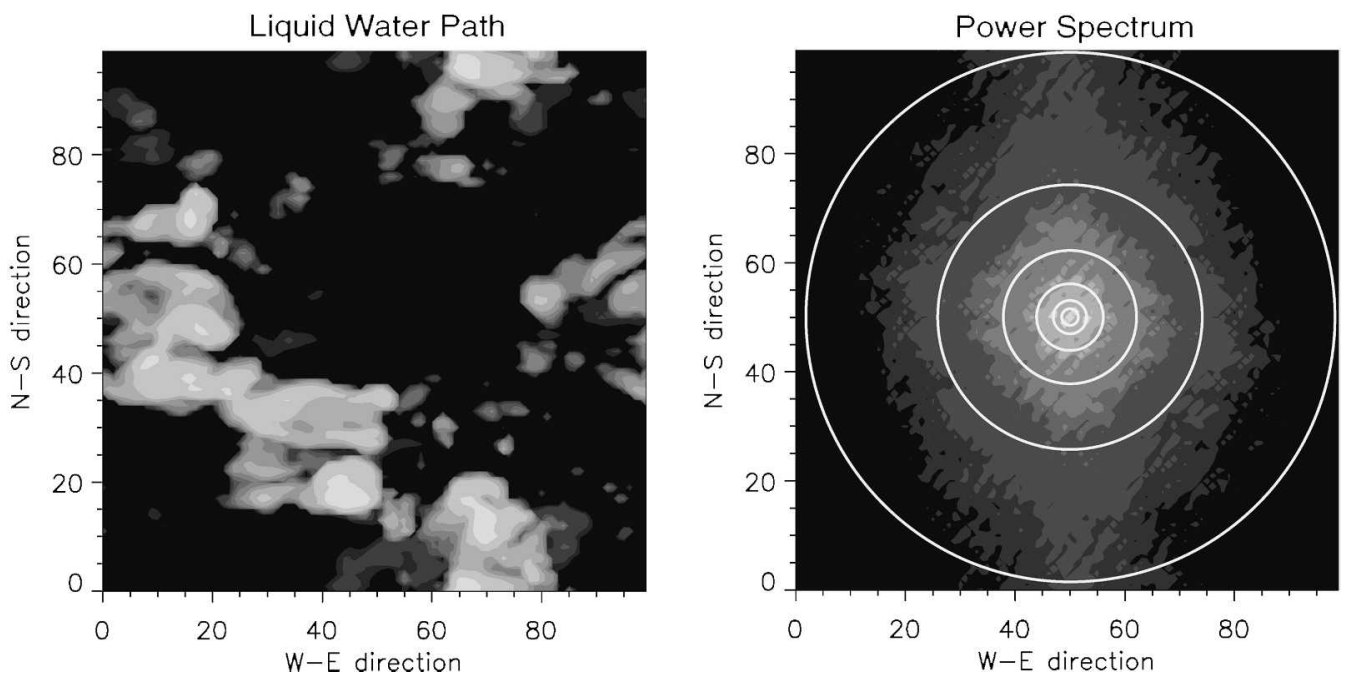

FIG. 1. Calculation geometry for the anisotropy parameter. (left) A sample liquid water path field exhibiting east-west alignment and (right) its power spectrum. The circles drawn on the power spectrum mark the boundaries of octave spatial frequency bands.

zontal and $45^{\circ}$ (diagonal) directions, respectively. Treating $M_{s, n}$ and $M_{c, n}$ as abscissa and ordinate values, the anisotropy parameter is calculated according to

$$
\left|A_{n}\right| \equiv \frac{\sqrt{M_{c, n}^{2}+M_{s, n}^{2}}}{M_{0, n}}
$$

and

$$
\angle\left(A_{n}\right) \equiv \frac{1}{2} \tan ^{-1}\left(\frac{M_{s, n}}{M_{c, n}}\right) .
$$

The magnitude of the anisotropy parameter will be zero if the original field is completely isotropic and one if the field is perfectly anisotropic (i.e., linear). All things being equal, a higher anisotropy parameter amplitude indicates greater anisotropy in the field. However, interpreting the magnitude of the anisotropy parameter at different spatial scales is complicated by the changing number of sample points per spectral band as spatial frequency $f$ increases. The fact that the total power in a band is spread over ever more points, so that the spectral density decreases as $f$ increases, is already accounted for by the normalization of $M_{c, n}$ and $M_{s, n}$ by $M_{0, n}$ in Eq. (4). However, it is more likely that anisotropy will occur by chance in a region containing fewer elements (here, the lower spatial frequency part of the spectrum) than in a region containing many elements (as in the high spatial frequency annuli). This problem has been addressed by devising a statistical test for the significance of calculated anisotropy levels. The general procedure for this test is to create a large ensemble of nominally isotropic but randomly varying instances of the type of field in question. After calculating the anisotropy parameter for all members of the ensemble, the probability density function of the resulting AP amplitudes is plotted for each spatial frequency range. The values at the 90th or 95th percentile of the distributions are considered the AP significance thresholds for this type of field. The significance criteria for anisotropy in various types of fields will be presented as needed below. In each case, the significance criteria decrease with increasing spatial frequency, reflecting the greater number of degrees of freedom in the data fields at small scales.

The angle obtained from Eq. (5) is expressed with respect to frequency space according to mathematical conventions. This angle must be rotated by $90^{\circ}$ to be referenced to real space and then converted to the meteorological angle convention. At this point, the resulting angle is interpreted as the predominant direction (long axis) of orientation of the structure in the field at the given scale. Note, however, that, depending on the size of the field elements with respect to the spatial scale under consideration, the anisotropy detected may be due to the shape of the elements themselves, their grouping and alignment, or some combination of these factors.

Application of the anisotropy parameter to idealized sample fields is illustrated by Fig. 2 and Table 1. These cases were selected to demonstrate the ability of the anisotropy parameter to detect different types of spatial organization. For example, the ellipse in Fig. 2a is clearly oriented $45^{\circ}$ to the left of vertical. The anisotropy parameter indicates alignment at a meteorological angle of $315^{\circ}$. While this angle is constant with spatial frequency, the strength of the measured anisotropy decreases at lower frequencies (larger scales) as the amplitude of the ellipse falls off relative to the background. Anisotropy changes more obviously with scale 

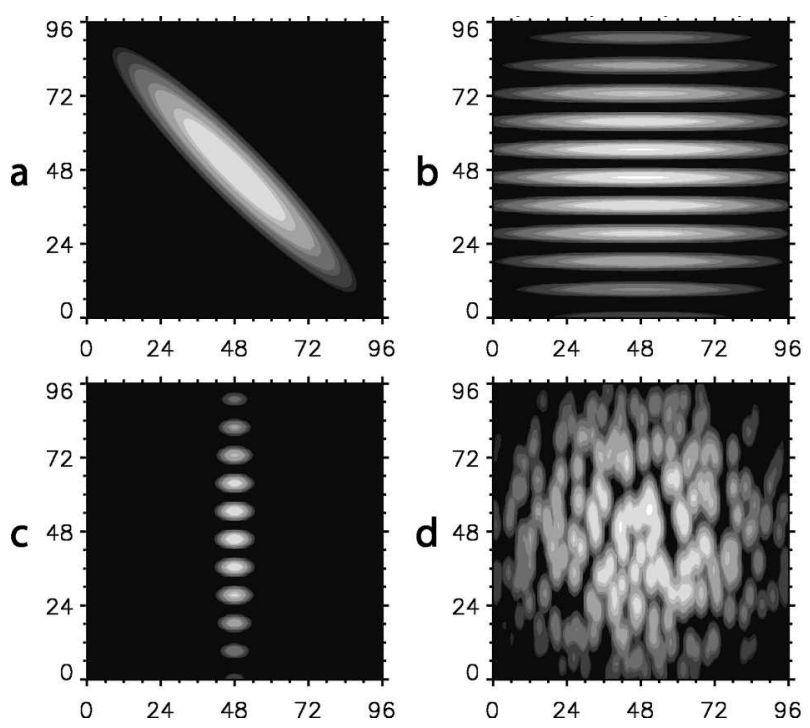

FIG. 2. Sample two-dimensional fields shown on a logarithmic scale.

in the other examples. For instance, a column of horizontal ellipses with circular weighting appears in Fig. $2 \mathrm{~b}$. Though the anisotropy parameter indicates strong horizontal alignment at scales smaller than 16 grid units, the AP amplitude falls off to zero and the angle becomes meaningless above 16 grid units due to the axial symmetry of the image at these scales. The remaining examples can be interpreted similarly. Note that significance values are not relevant to these idealized cases.

Power spectral analysis has been used to identify directionality and characteristic scales in two-dimensional fields in other disciplines for some years (Pincus and Dobrin 1966; Renshaw and Ford 1984; Prince and Ehrlich 1990; Derbyshire et al. 1992; Peng and Kirk 1997; Couteron and Lejeune 2001) and has recently been applied to the study of mesoscale cloud structure (Wood and Hartmann 2001). Determining these properties directly from an image of a field is tedious and requires measuring the size and orientation of each image feature individually. Obtaining these values from the power spectrum of a data field is much simpler because, in the data transformation, positional information is lost in favor of representation in terms of spatial frequency and direction. Using the FFT to calculate the power spectrum makes this process nearly instantaneous on modern computers. Earlier, simple visual inspection was used to select the direction of greatest prominence in the power spectrum (Pincus and Dobrin 1966), but now most authors choose to analyze the spectrum as a function of frequency to obtain the dominant scale and as a function of angle to find the primary orientation (Renshaw and Ford 1984; Prince and Ehrlich 1990; Peng and Kirk 1997; Couteron and Lejeune 2001; Wood and Hartmann 2001). However, the identified direction of orientation may not occur at the computed dominant scale. The anisotropy measure defined here is unique in that it permits the orientation of structures to be determined as a function of spatial frequency.

\section{b. Tilt}

We define vertical tilt as the amount of horizontal shift in the cloud field structure per unit height. In this case, the vertical increments are naturally determined by the vertical grid spacing of the model cloud fields. The horizontal displacement between structures in neighboring liquid water content planes is obtained by cross-correlating the two arrays and then locating the peak of their two-dimensional cross-correlation function. We compute the cross-correlation functions via Fourier methods (Press et al. 1992). During this process, Fourier interpolation, that is, increasing the array size in the Fourier domain by adding zeroes at frequencies above the resolution of the original data, is applied. This allows the correlation peaks to be located with precision better than the grid spacing of the modeled cloud fields.

The tilt calculation technique is illustrated in Fig. 3 using liquid water content (LWC) fields from levels 32 and 35 in a scene from a large-eddy simulation with high shear (SU10). This particular scene occurs $7.75 \mathrm{~h}$ into the simulation, about $2 \mathrm{~h}$ after the cumulus clouds have formed. The input scenes are shown to the left and the full cross-correlation function is in the center. The enlarged image of the correlation function to the right shows the cross-correlation peak displaced 0.94 grid cells to the east and 0.33 toward the south. Taking into

TABLE 1. Anisotropy parameter values for test images of Fig. 2. Scales are given in number of grid units in Fig. 2.

\begin{tabular}{|c|c|c|c|c|c|c|c|c|c|c|}
\hline Band edges & & 48 & 33 & 24 & 16 & 12 & 8 & 6 & 4 & 3 \\
\hline $\mathrm{a}$ & $\begin{array}{l}\text { Amplitude } \\
\text { Angle }\end{array}$ & $\begin{array}{l}0.783 \\
315^{\circ}\end{array}$ & $\begin{array}{l}0.866 \\
315^{\circ}\end{array}$ & $\begin{array}{l}0.925 \\
315^{\circ}\end{array}$ & $\begin{array}{l}0.974 \\
315^{\circ}\end{array}$ & $\begin{array}{l}0.980 \\
315^{\circ}\end{array}$ & $\begin{array}{l}0.992 \\
315^{\circ}\end{array}$ & $\begin{array}{l}0.994 \\
315^{\circ}\end{array}$ & $\begin{array}{l}0.998 \\
315^{\circ}\end{array}$ & $\begin{array}{l}0.997 \\
315^{\circ}\end{array}$ \\
\hline b & $\begin{array}{l}\text { Amplitude } \\
\text { Angle }\end{array}$ & $\begin{array}{l}0.000 \\
270^{\circ}\end{array}$ & $\begin{array}{l}0.001 \\
180^{\circ}\end{array}$ & $\begin{array}{l}0.055 \\
270^{\circ}\end{array}$ & $\begin{array}{l}0.979 \\
270^{\circ}\end{array}$ & $\begin{array}{l}0.988 \\
270^{\circ}\end{array}$ & $\begin{array}{l}0.983 \\
270^{\circ}\end{array}$ & $\begin{array}{l}0.997 \\
270^{\circ}\end{array}$ & $\begin{array}{l}0.998 \\
270^{\circ}\end{array}$ & $\begin{array}{c}0.999 \\
270^{\circ}\end{array}$ \\
\hline $\mathrm{c}$ & $\begin{array}{l}\text { Amplitude } \\
\text { Angle }\end{array}$ & $\begin{array}{l}0.668 \\
360^{\circ}\end{array}$ & $\begin{array}{l}0.866 \\
360^{\circ}\end{array}$ & $\begin{array}{l}0.946 \\
360^{\circ}\end{array}$ & $\begin{array}{l}0.947 \\
360^{\circ}\end{array}$ & $\begin{array}{l}0.548 \\
270^{\circ}\end{array}$ & $\begin{array}{l}0.230 \\
270^{\circ}\end{array}$ & $\begin{array}{l}0.665 \\
270^{\circ}\end{array}$ & $\begin{array}{l}0.746 \\
270^{\circ}\end{array}$ & $\begin{array}{l}0.874 \\
270^{\circ}\end{array}$ \\
\hline d & $\begin{array}{l}\text { Amplitude } \\
\text { Angle }\end{array}$ & $\begin{array}{l}0.278 \\
326^{\circ}\end{array}$ & $\begin{array}{l}0.500 \\
250^{\circ}\end{array}$ & $\begin{array}{c}0.211 \\
250^{\circ}\end{array}$ & $\begin{array}{c}0.244 \\
190^{\circ}\end{array}$ & $\begin{array}{c}0.405 \\
191^{\circ}\end{array}$ & $183^{\circ}$ & $\begin{array}{l}0.816 \\
356^{\circ}\end{array}$ & $\begin{array}{l}0.903 \\
358^{\circ}\end{array}$ & $\begin{array}{l}0.948 \\
360^{\circ}\end{array}$ \\
\hline
\end{tabular}




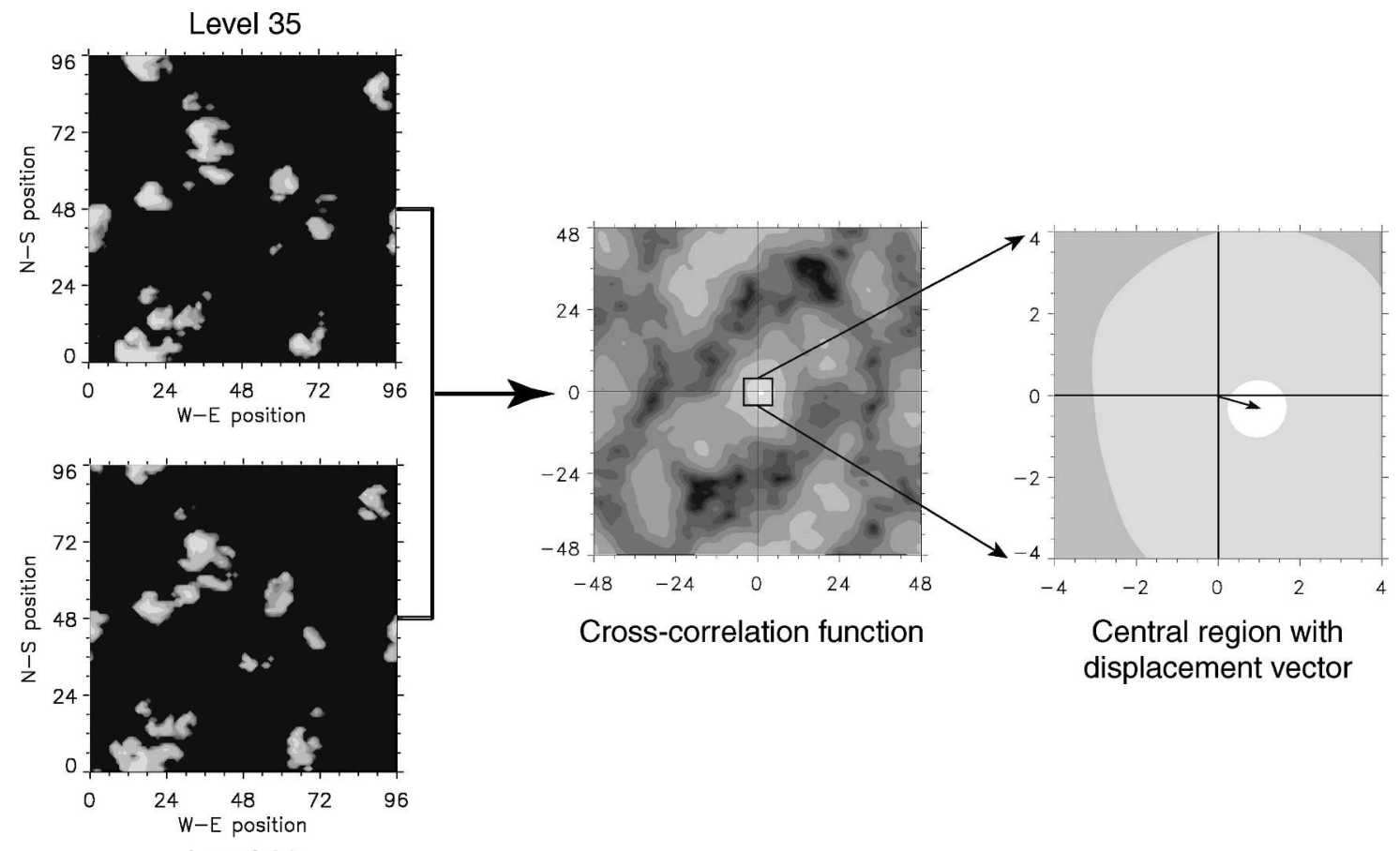

Level 32

FIG. 3. Schematic of tilt calculation method. The two-dimensional LWC fields are cross correlated. The displacement of the cross-correlation maximum from the origin indicates the position of the structures in the second LWC plane (level 35) relative to their location in the first LWC plane (level 32).

account the size of the grid boxes $(66.6667 \mathrm{~m})$, the upper field is displaced $66.0 \mathrm{~m}$ in the meteorological direction of $289^{\circ}$ relative to the lower field. Since the vertical grid spacing is $40 \mathrm{~m}$ and the planes are three levels apart, the tilt is $0.55 \mathrm{~m} \mathrm{~m}^{-1}$ or about $29^{\circ}$ from the vertical.

Liquid water content planes separated by three vertical grid spaces were used to illustrate the tilt calculation method because this yielded a displacement large enough to be visible in the accompanying figure. However, fields at adjacent heights are generally better correlated. Thus, for greater accuracy, throughout this work we calculate the tilt between neighboring LWC planes and average the results over the entire depth of the cloud field to obtain a single tilt value for each scene.

\section{Numerical modeling}

As discussed above, numerical cloud simulations were considered the optimum method to examine the meteorological conditions that give rise to anisotropy in fair-weather cumulus cloud fields. Since interactions between cloud particles and radiation occur on scales of tens of meters, a model with resolution on this order and a domain size of several kilometers is appropriate for this work. In addition, aspects of the boundary layer that influence cloud structure, particularly turbulent motions, should be represented as accurately as reasonably possible.

Both large-eddy simulation (LES) and direct numerical simulation (DNS) models of the moist atmosphere meet these criteria. However, the subgrid model in an LES turns off in regions where a local measure of the Richardson number is small, which inhibits small-scale mixing in the quiescent portions of the cloud free environment. This helps the LES to maintain a more realistic cloud layer structure than would occur for DNS, although at the cost of a less defensibly physical treatment of the small scales.

Since realistic structure in the simulated cloud fields is essential for this study, we elected to employ a largeeddy simulation model. A base case in which fairweather cumulus develop was first established, then modified versions of this case were run. The environmental conditions most conducive to the development of anisotropy in the modeled cloud fields were then determined by analyzing the structure of the modeled cloud fields.

\section{a. UCLA model}

The University of California, Los Angeles (UCLA) LES model, a three-dimensional, finite-difference flow solver described by Stevens et al. (1999), was selected for use in this study. This code has been thoroughly evaluated in a number of model comparisons (Brether- 
ton et al. 1999; Stevens et al. 2001; Brown et al. 2002) and provides a state-of-the-art representation of the turbulent structure in nonprecipitating cloud-topped atmospheric boundary layers. Because the details of this model have been presented elsewhere, only information specific to this work is given here.

The UCLA LES model incorporates a first-order Smagorinsky-type subfilter-scale model. Turbulence was initiated by adding random potential temperature deviations to the lowest levels of the atmosphere at model initialization. The seed of the random number generator used to supply the perturbation values could be set by the user. This allowed repeated simulations to begin with identical potential temperature deviation fields while other conditions varied, removing one variable from consideration.

For the simulations reported here, different boundary conditions were applied at the various edges of the computational domain. Periodic boundary conditions were used in the horizontal directions, making it unnecessary to provide values at the boundaries after runs were started. Rayleigh friction was applied to the top ten model layers to prevent waves from reflecting from the top of the domain. The bottom surface had no topography and was assigned a roughness length of $0.035 \mathrm{~m}$ to represent tall grass. No-slip conditions were applied at this surface.

Because of the cyclic boundary conditions, there was no advection into or out of the modeled volume. As a result, large-scale atmospheric changes caused by synoptic-scale patterns needed to be artificially imposed on the domain. In these large-eddy simulations, moisture and temperature advection over time were specified in a subroutine and communicated to the system as additions to the moisture and temperature tendency terms.

Clouds were represented in the model only in terms of the liquid water mixing ratio. No bulk or microphysical parameterization was employed. Total mixing ratio was calculated explicitly and the liquid portion was diagnosed based on the local saturation mixing ratio. Using a simple all-or-nothing saturation scheme allows us to focus on the dynamical processes of the simulated system without the added complexity of cloud water parameterization. However, since rain cannot occur in this model, the dynamical feedback associated with rain is neglected. Exactly how the development of precipitation may affect anisotropy is thus left an interesting open question.

Radiative processes were not explicitly computed in these simulations. Instead, large-scale atmospheric heating rate profiles were prescribed as a function of time. The effect of radiation at the surface was incorporated by specifying the resulting surface latent and sensible heat fluxes, also as a function of time. These simplifications substantially reduced the run time of the model and eliminated the need for a surface model but precluded the interaction of clouds and radiation. This

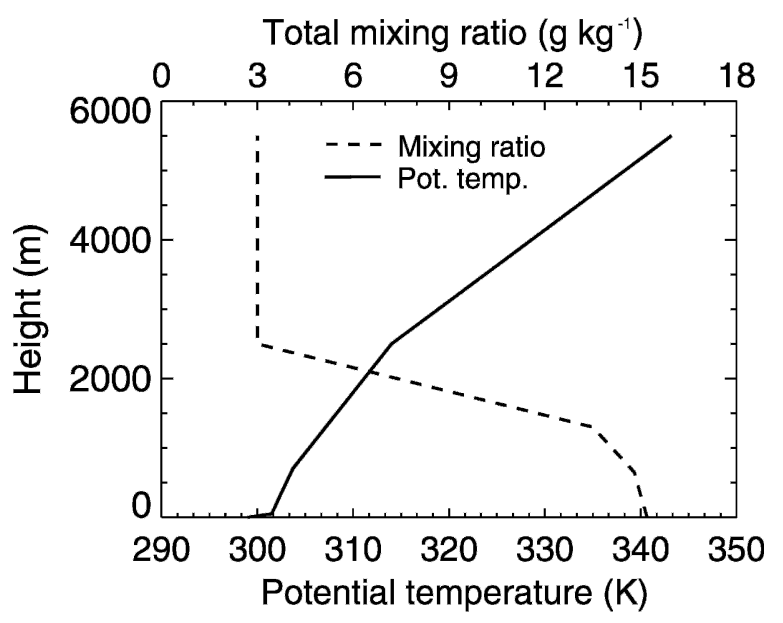

FIG. 4. Initial sounding used for the large-eddy simulations.

decoupling, while unrealistic, has been found to have little effect on the simulation (Brown et al. 2002) and has the advantage of removing another source of variability from the model, so that the imposed environmental changes are the primary cause of the observed differences between runs.

\section{b. LES cases}

\section{1) Base case details}

Case six of the Global Energy and Water Cycle Experiment (GEWEX) Cloud System Study (GCSS) Working Group 1 (Brown et al. 2002) was used as the base large-eddy simulation scenario. This case, developed for an LES comparison project, simulates the formation of cumulus clouds over the Department of Energy Atmospheric Radiation Measurement (ARM) program's Southern Great Plains (SGP) site. In this case, a continental boundary layer is forced by the diurnal cycle. The initial atmospheric thermodynamic state, illustrated in Fig. 4, was based on radiosonde measurements made at the ARM Southern Great Plains site central facility on 21 June 1997, with some adjustments. The maximum amplitude of the initial potential temperature deviations added to this profile to initiate turbulence was $0.1 \mathrm{~K}$ at the surface and decreased linearly with height to $0.0 \mathrm{~K}$ at $200 \mathrm{~m}$. A uniform westerly wind at $10 \mathrm{~m} \mathrm{~s}^{-1}$ was imposed throughout the model domain at the start of the simulation. This wind profile is a simplification of the winds observed by the central facility radiosondes. Diurnal forcing was incorporated by specifying time-varying sensible and latent heat fluxes at the surface, as shown in Fig. 5. The surface fluxes were determined by fitting the measured heat fluxes from the SGP site with piecewiselinear functions. Both fluxes increase until early afternoon and decrease in the evening. The latent heat flux 


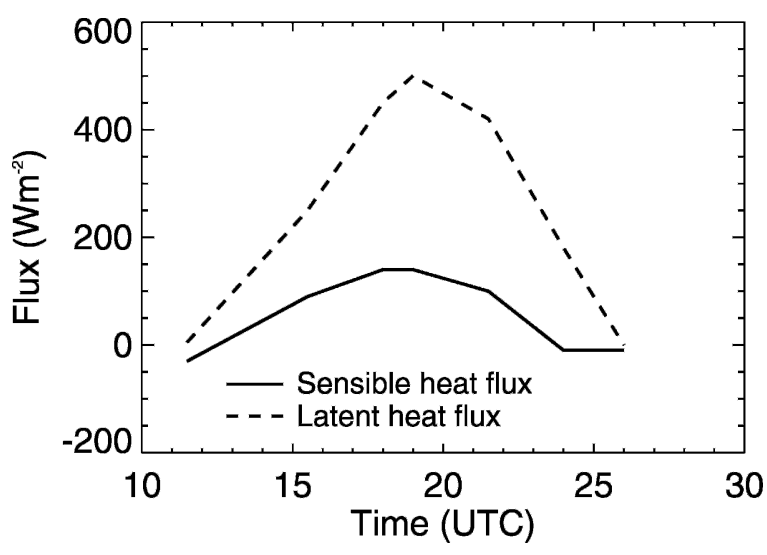

FIG. 5. Surface fluxes for standard large-eddy simulation run.

is the dominant of the two energy sources, with Bowen ratios falling as low as 0.28 during the afternoon. The prescribed large-scale tendencies, shown in Fig. 6, are relatively small. The large-scale advective tendencies over time were derived from observations using a varia-
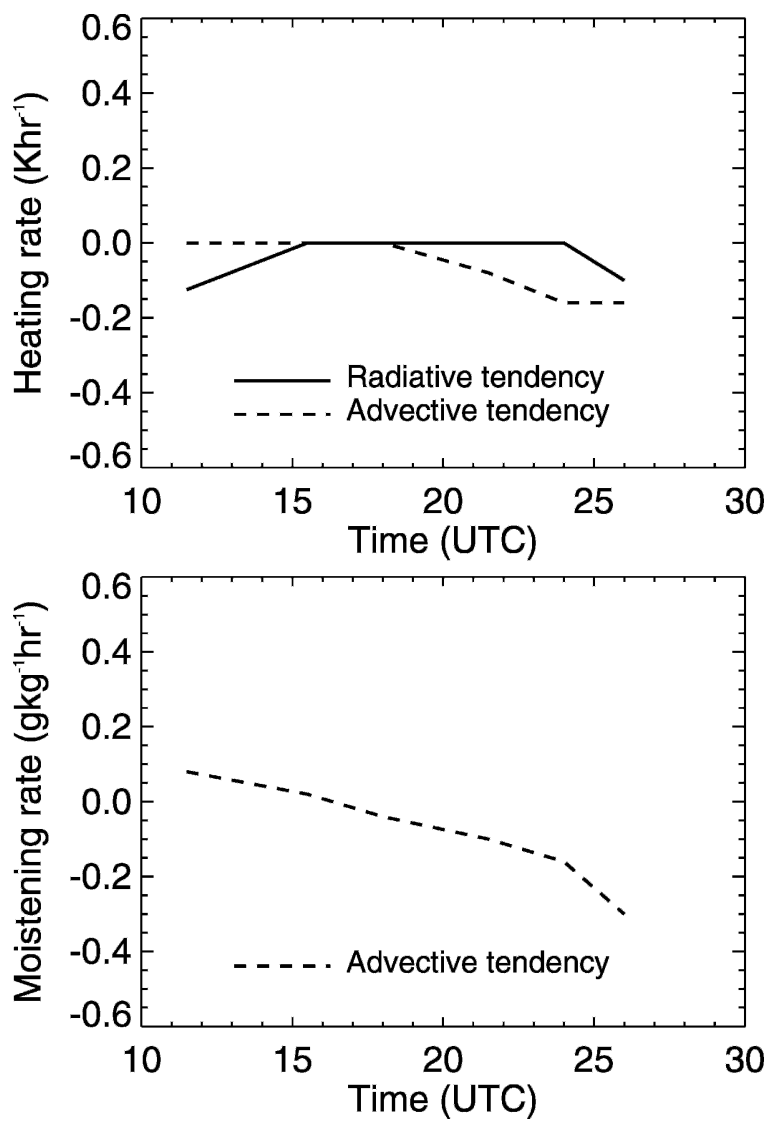

FIG. 6. Maximum values of large-scale forcings for standard large-eddy simulation run. These values were used from the surface up to $1000 \mathrm{~m}$. The values were reduced linearly to zero from 1000 to $2000 \mathrm{~m}$. Above $2000 \mathrm{~m}$, no large-scale forcings were applied. tional method (Zhang et al. 2001), while the radiative tendencies were diagnosed by running a simulation that included two-stream radiative transfer computations. The net large-scale radiative tendency is a slight cooling in the morning and evening. Advection causes cooling in the afternoon and evening along with moistening in the morning and drying during the rest of the simulation. Together, the imposed conditions produce radiatively forced development of shallow cumulus, with weak large-scale forcing. The results from the UCLA model were similar to those from the other LES models in the GCSS comparison study (Brown et al. 2002).

The model configuration used here had a $96 \times 96 \times$ 110 element grid with $66.6667 \times 66.6667 \times 40.00 \mathrm{~m}^{3}$ spacing. Simulations began at 0530 local time and were run out $12 \mathrm{~h}$ with a base time step of 1.0 to $1.5 \mathrm{~s}$, depending on stability conditions. Three-dimensional model outputs, such as mixing ratio and velocity fields, were saved every $15 \mathrm{~min}$ after clouds began to form, which occurred about $5 \mathrm{~h}$ into the run, while integrated profiles were written out every $30 \mathrm{~min}$. Five instances of the base case, each with different initial random temperature variations, were run to establish the range of results that could be expected from the model for a single scenario.

\section{2) Modified LES RUNS}

The influence of the following environmental conditions on cloud field structure was tested by means of additional LES runs: wind speed, wind shear, amount of available water vapor, and the magnitude of surface fluxes. The simulations are summarized in Table 2. Modifications to the base case made to test these effects are coded into the run names as follows: UN indicates uniform westerly initial winds with the speed indicated in $\mathrm{m} \mathrm{s}^{-1}$; SUN indicates an initial vertical shear in the boundary layer with westerly winds increasing with height to a maximum of $N \mathrm{~m} \mathrm{~s}^{-1}$ at $2500 \mathrm{~m}$, while SVN is the same but for a southerly speed shear; BLWP indicates that the available water vapor in the boundary layer has been set to the given percentage of the standard amount; and FP indicates that the surface fluxes have been set to the given percentage of the base case values. As shown in the table, unless other zonal winds were specified, the initial uniform $10 \mathrm{~m} \mathrm{~s}^{-1}$ westerly wind of the base case was used as an initial condition in all runs.

The environmental variations imposed in the different model runs were selected from those to which cloud deformation or organization were attributed in the literature, as discussed above. Horizontal winds and wind shear were explicitly mentioned in the prior work. Changes in the surface forcing and initial water vapor profile were included as ways of influencing buoyancy. The magnitudes of the variations applied were designed to span a range reasonable for cumulus clouds.

As in the base case, all simulations started at 0530 
TABLE 2. Summary of large-eddy boundary layer cumulus simulation cases. Boundary layer $(0-2500 \mathrm{~m})$ winds and water vapor variations are imposed as initial conditions while surface fluxes are specified throughout the runs.

\begin{tabular}{|c|c|c|c|}
\hline Case & Steady wind & Vertical wind shear & Other conditions \\
\hline U0 & $0 \mathrm{~m} \mathrm{~s}^{-1} @ 270^{\circ}$ & - & - \\
\hline U5 & $5 \mathrm{~m} \mathrm{~s}^{-1} @ 270^{\circ}$ & - & - \\
\hline U10* & $10 \mathrm{~m} \mathrm{~s}^{-1} @ 270^{\circ}$ & - & - \\
\hline U15 & $15 \mathrm{~m} \mathrm{~s}^{-1} @ 270^{\circ}$ & - & - \\
\hline $\mathrm{U} 20$ & $20 \mathrm{~m} \mathrm{~s}^{-1} @ 270^{\circ}$ & - & - \\
\hline SU5 & $0 \mathrm{~m} \mathrm{~s}^{-1} @ 270^{\circ}$ & $2 \mathrm{~m} \mathrm{~s}^{-1} \mathrm{~km}^{-1} @ 270^{\circ}$ & - \\
\hline SU10 & $0 \mathrm{~m} \mathrm{~s}^{-1} @ 270^{\circ}$ & $4 \mathrm{~m} \mathrm{~s}^{-1} \mathrm{~km}^{-1} @ 270^{\circ}$ & - \\
\hline SV5 & $10 \mathrm{~m} \mathrm{~s}^{-1} @ 270^{\circ}$ & $2 \mathrm{~m} \mathrm{~s}^{-1} \mathrm{~km}^{-1} @ 180^{\circ}$ & - \\
\hline SV10 & $10 \mathrm{~m} \mathrm{~s}^{-1} @ 270^{\circ}$ & $4 \mathrm{~m} \mathrm{~s}^{-1} \mathrm{~km}^{-1} @ 180^{\circ}$ & - \\
\hline F50 & $10 \mathrm{~m} \mathrm{~s}^{-1} @ 270^{\circ}$ & - & Surface fluxes at $50 \%$ \\
\hline $\mathrm{F} 150$ & $10 \mathrm{~m} \mathrm{~s}^{-1} @ 270^{\circ}$ & - & Surface fluxes at $150 \%$ \\
\hline BLW090 & $10 \mathrm{~m} \mathrm{~s}^{-1} @ 270^{\circ}$ & - & $-10 \%$ available water in boundary layer \\
\hline BLW110 & $10 \mathrm{~m} \mathrm{~s}^{-1} @ 270^{\circ}$ & - & $+10 \%$ available water in boundary layer \\
\hline
\end{tabular}

* This is the base case.

local time and ended at 1730. The output saved was also identical. Examples of the simulated cloud fields appear in Fig. 7. Varying degrees of tilt and horizontal anisotropy are evident in these scenes.

\section{Anisotropy and tilt analysis}

\section{a. Method}

Horizontal anisotropy in the liquid water content fields from the large-eddy simulations was evaluated using the anisotropy parameter described in section $2 \mathrm{a}$. To reliably capture the anisotropy trends associated with the various environmental conditions, multiple fields from each LES run were analyzed. The first ten scenes with a cloud fraction between 0.20 and 0.35 from each run were selected for study. This criterion ensured that there were ample clouds in each scene considered and that all the scenes were relatively similar aside from the changing forcings.

The anisotropy parameter could be calculated for each horizontal slice through the three-dimensional liquid water content fields individually. Instead, averaging was performed over all the selected data for each run. First, the two-dimensional power spectra computed for all the slices in a scene were averaged together. The resulting spectra were then averaged over the ten scenes selected for the LES run. The anisotropy parameter was computed for the final average spectrum. Besides providing more robust results, this form of averaging has the added benefit of inherently weighting the input spectra according to the amount of liquid water in each layer.

The significance of the anisotropy parameter magnitudes calculated for the averaged liquid water content spectra was estimated using an ensemble of $10000 \mathrm{cu}-$ mulus cloud scenes produced using the stochastic field generation technique described in Evans and Wis- combe (2004) modified to take three-dimensional cloud data as input. The fields were based on statistics from 12 LES cloud scenes drawn from the base case runs. This forced the stochastic fields to have the same average spectral characteristics as the LES fields. The 10000 scenes were divided into groups of ten and the LWC anisotropy calculation was performed for each group in the manner described above for the LES scenes. Histograms of the resulting 1000 anisotropy parameter values for each spatial frequency band were plotted and the 50th, 90th, and 95th percentiles were found. Since the stochastic field generation algorithm is based on isotropically averaged input power spectra multiplied by random values, these percentiles indicate the likelihood of anisotropy being produced by random chance in otherwise isotropic fields similar to those from the LES. The significance values computed in this way are shown in Table 3.

Tilt detection was also performed on the ten selected scenes from each of the LES runs. For a given scene, the displacement between adjacent horizontal liquid water content slices with cloud fractions greater than $5 \%$ was determined by locating the peak of their twodimensional cross-correlation function, as detailed in section $2 \mathrm{~b}$. The detected displacements were averaged over the vertical extent of the given liquid water content field and then over all ten scenes selected for that LES run. The original liquid water content slices were on $96 \times 96$ grids with $66.6667 \mathrm{~m}$ grid spacing, but during the computation, they were interpolated to $2048 \times 2048$ grids at a spacing of $3.1 \mathrm{~m}$. This means that the smallest resolvable tilt between two levels was $3.1 \mathrm{~m}$ per $40.0 \mathrm{~m}$ of rise or $0.0775 \mathrm{~m} \mathrm{~m}^{-1}$ ( $4.44^{\circ}$ from the vertical).

The significance of the resulting tilt values was estimated by performing identical calculations for 2000 stochastic cloud scenes. Significance values based on the statistics from 200 groups of ten stochastic scenes are listed in Table 4. Note that these values are very small, 

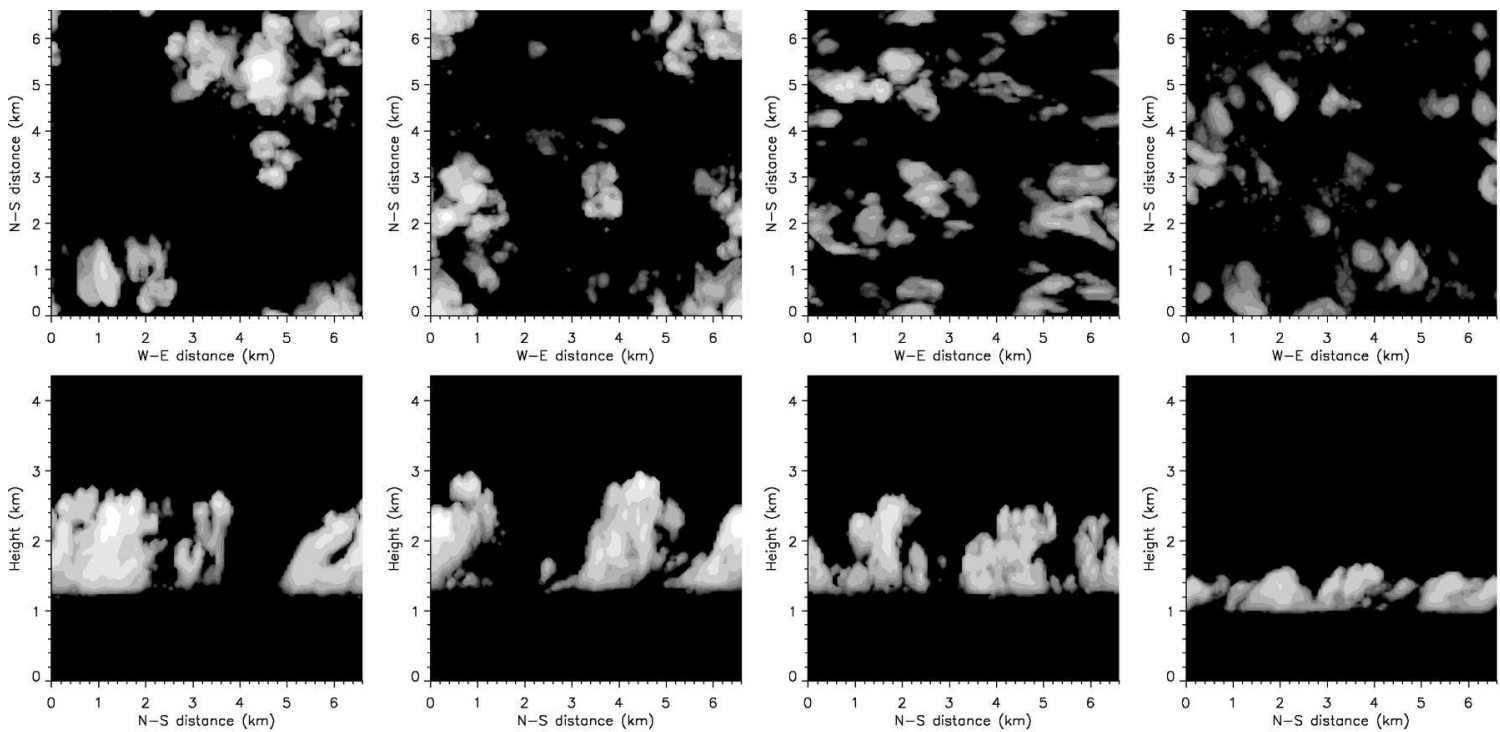

500

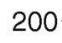

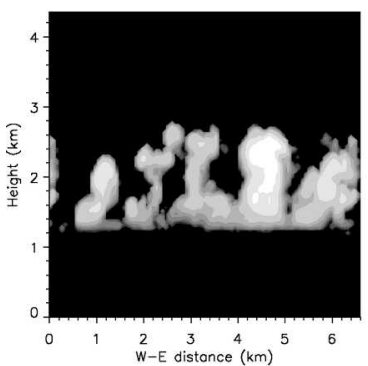

U10

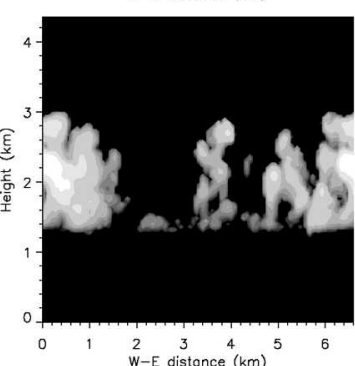

BLW110

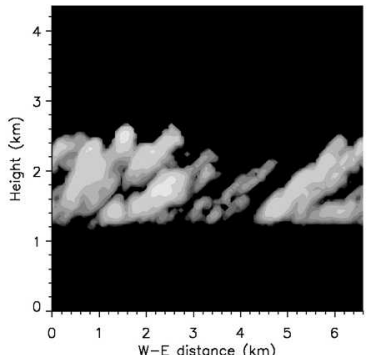

SU10

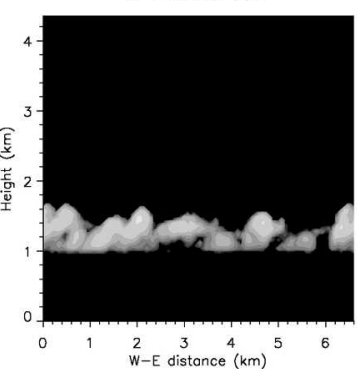

F50

FIG. 7. Sample cloud fields from large-eddy simulations. Snapshots of integratred LWC are shown from the top, west, and south.

All images are for $10 \mathrm{~h}$ into the model run.

indicating that tilt is unlikely to occur by random chance since coordinated movements at many points are required to create tilted structures.

\section{b. Results}

\section{1) Horizontal anisotropy AS A FUnCtion OF} SPATIAL FREQUENCY

Results of the liquid water content anisotropy parameter calculations are presented as a function of scale in Fig. 8. In Fig. 8a, results for five instances of the base case are shown. Dynamically, these cases are identical; only their initial random potential temperature perturbations differ. Taken together, they demonstrate the natural range of variability in the LES runs. Deviations of the order shown might thus be expected for multiple runs of the other modeled scenarios. Notice that the anisotropy measured for the base cases is generally not significant, although it does approach significance for the highest spatial frequencies.

Results for the five cases having uniform initial wind profiles are shown in Fig. 8b. The initial wind speeds in these cases range from 0 to $20 \mathrm{~m} \mathrm{~s}^{-1}$. At most scales, the detected anisotropy is insignificant. However, significant anisotropy does occur at higher frequencies for the 15 and $20 \mathrm{~m} \mathrm{~s}^{-1}$ runs, suggesting that uniform winds can cause anisotropy at small scales in cumulus clouds. It should be pointed out however that, although no shear

TABLE 3. Significance values for anisotropy parameters calculated using the horizontal LWC fields for all heights in 10 scenes. All averaging was performed in the Fourier domain. Spatial frequencies are given in $\mathrm{m}^{-1}$.

\begin{tabular}{cccccccccrrr}
\hline \hline Band edges & $1 / 3200$ & \multicolumn{2}{c}{$1 / 2262$} & $1 / 1600$ & $1 / 1131$ & \multicolumn{1}{c}{$1 / 800$} & $1 / 565$ & $1 / 400$ & $1 / 282$ & $1 / 200$ & $1 / 141$ \\
\hline $50 \%$ & 0.079 & 0.074 & 0.068 & 0.058 & 0.046 & 0.032 & 0.027 & 0.018 & 0.011 \\
$90 \%$ & 0.134 & 0.130 & 0.115 & 0.097 & 0.077 & 0.054 & 0.043 & 0.029 & 0.019 & 0.032 & 0.022 \\
$95 \%$ & 0.150 & 0.149 & 0.128 & 0.109 & 0.086 & 0.061 & 0.048 & 0.032 \\
\hline
\end{tabular}


TABLE 4. Tilt significance values for ten scenes averaged together.

\begin{tabular}{cc}
\hline \hline Significance level & Tilt $\left(\mathrm{m} \mathrm{m}^{-1}\right)$ \\
\hline $50 \%$ & 0.0156 \\
$90 \%$ & 0.0234 \\
$95 \%$ & 0.0313 \\
\hline
\end{tabular}

was imposed on these runs, shear does develop because of interaction between the specified winds and friction at the lower surface. From this plot, it is not possible to say whether the detected anisotropy is due to the developing vertical speed shears or simply to the presence of the winds themselves. This question will be considered further in section $4 b(4)$.

In Fig. 8c, two cases with imposed vertical shears are compared to the average of the base cases. In the initially sheared wind fields, the horizontal wind is zero at the surface and increases at the rates indicated in Table 2. In both of these cases, the horizontal anisotropy in the output cloud fields is noticeably greater than for the base case and well above the $95 \%$ significance level. The anisotropy occurs at moderate as well as high spatial frequencies. The degree of anisotropy increases with the strength of the shear, as might be expected.

Some anisotropy is evident at the higher frequencies in the sheared cases depicted in Fig. 8d. In these cases, the speed shear was imposed perpendicular to the standard uniform wind and directional shear results. This makes it difficult to discern the effect of the shear on cloud field structure. If the increasing wind speeds do cause distortion of the cloud structure, the direction of the anisotropy will likely change with height. Averaging the liquid water content spectra over height will then smear out any structure, reducing the measured anisotropy. This smearing effect should be greater in case SV10 than in SV5 since the direction of the wind changes more rapidly with height in this case. Despite the application of vertical averaging, some degree of anisotropy is detected for both cases at spatial frequencies above $1 / 400 \mathrm{~m}$. As expected, the anisotropy values for SV10 are lower than for SV5 at most scales.

The results in Fig. 8e indicate that cloud field anisotropy is greater when the magnitude of surface forcing is lower. This is not an unexpected finding. Competition between shear and buoyant forces has long been considered a factor in mesoscale atmospheric organization (LeMone 1973; Grossman 1982; Shirer 1986; Sykes and Henn 1989; Etling and Brown 1993; Weckwerth et al. 1997; Glendening 2000) because strong convection disrupts the ability of shear to organize wind flow patterns. In this case, we find significant anisotropy at most scales when surface forcing is decreased by a factor of two, while the cases with standard or increased forcing are not significantly anisotropic.

A comparison of the horizontal anisotropy of cumu- lus cloud scenes from simulations in which the initial water vapor profile was varied is shown in Fig. 8f. Because the clouds formed in the presence of lower water vapor concentrations are small and sparse, only four scenes from the reduced water vapor run met the usual cloud fraction criterion for analysis. For this case only, the required cloud fraction was thus reduced to $15 \%$, yielding more than the ten scenes required for this analysis. Of course, not only the amount of cloud, but also the time required for its formation, was affected by the variations in humidity. The ten scenes analyzed for the high humidity case occurred much earlier in the simulation than the ten scenes from the low water vapor case. This difference in boundary layer development time may affect the comparison.

Based on the data shown, total available water vapor did not have a large impact on the anisotropy of the resulting cloud fields, except possibly at the very highest frequencies. It is conjectured that the effect of increased/decreased water vapor is similar to that of increased/decreased surface forcing, though smaller in magnitude, because the amount of energy made available by latent heat release during cloud formation is much smaller than that provided by the changes in surface forcing. In fact, because these are fair-weather cumulus clouds, the total latent heat release amounts to less than $1 \%$ of the surface forcing over the period of cloud formation, but this heating is concentrated in the regions of cloud formation.

In each of the cases evincing anisotropy, the anisotropy is most pronounced at high spatial frequencies in contrast to standard turbulence theory, which holds that turbulent structures become more isotropic at smaller scales. Viewed as a lack of anisotropy at large scales, this trend may be the result of limitations of the simulated fluid dynamics due to domain size and the periodic boundary conditions. Alternatively, environmental conditions not included in this study, such as the inflection-point instability or parallel instability that are important to the formation of roll clouds (Etling and Brown 1993), may be required for the formation of large-scale oriented cloud structures. Another possible explanation is that the scales of anisotropy are related to the characteristic scales of the dynamical system and the obvious characteristic length scales here-cloud layer depth and subcloud layer depth-are both relatively small (about 2000 and $1000 \mathrm{~m}$, respectively). A final possibility is that the excessive small-scale anisotropy observed here is due to poor representation of the small-scale flow by the model. This possibility is discussed further in section 5 .

\section{2) Overall HORIZONTAL ANISOTROPY}

Horizontal anisotropy results averaged over all frequency bands are presented in Table 5. These values were obtained by vector addition of the anisotropy parameters computed for the nine half-octave intervals 

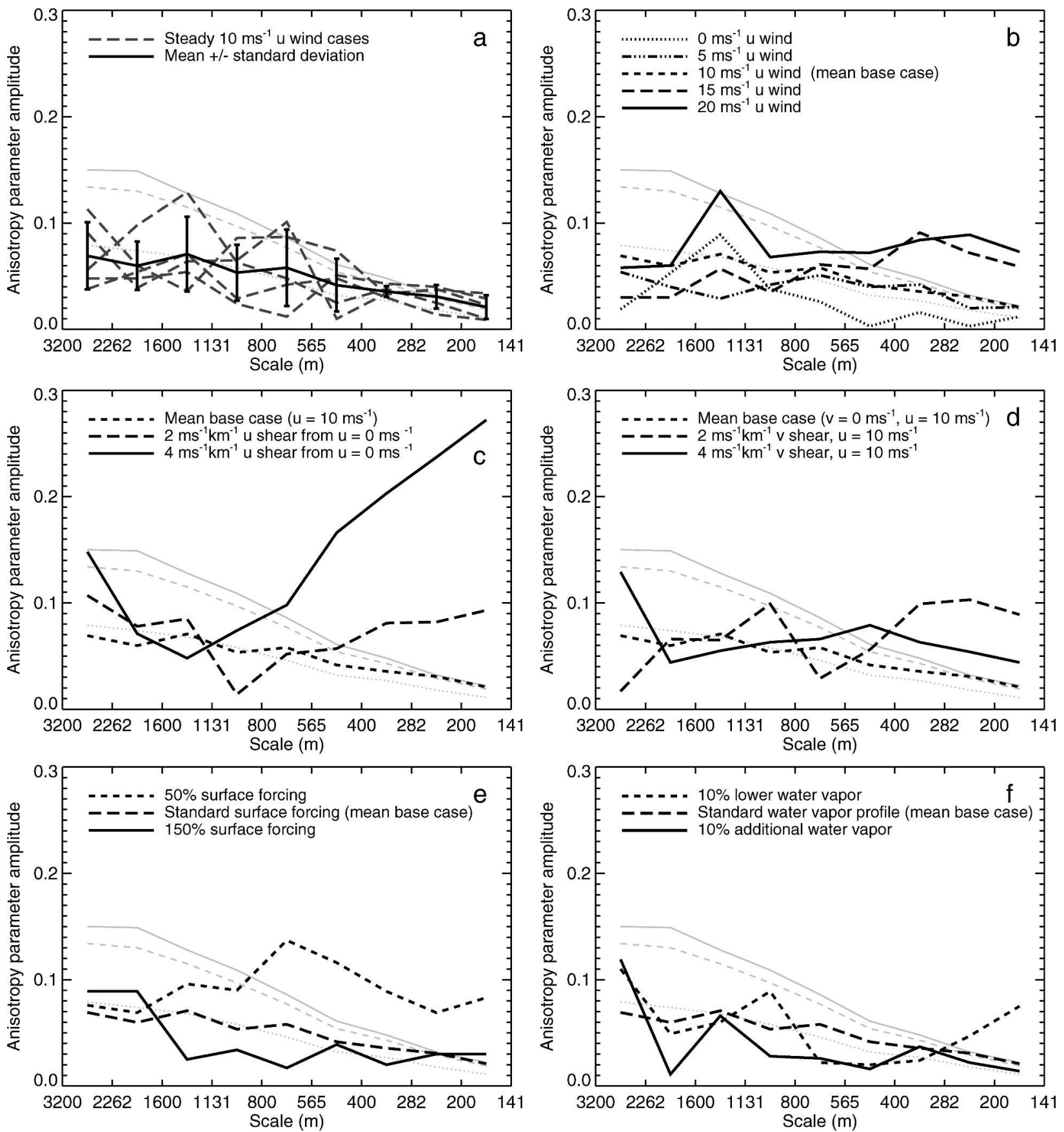

FIG. 8. LWC anisotropy magnitudes calculated for LES clouds generated with various environmental conditions. (a) Base case runs with different initial random potential temperature variations. (b) Uniform initial wind profile cases. (c) Vertical speed shear cases. (d) Directional shear cases. (e) Cases with modified surface forcing. (f) Cases with modified water vapor concentrations. The narrow gray dotted, dashed, and solid lines are the 50\%, 90\%, and 95\% significance levels, respectively.

from $1 / 3200 \mathrm{~m}$ to $1 / 141 \mathrm{~m}$. Each anisotropy vector was weighted by the inverse of the variance of the anisotropy parameter amplitude calculated for the corresponding spatial frequency interval in the significance test computations. This accounted for the degree of error expected in the anisotropy vectors at each spatial frequency due to random chance so that less significant values were given less weight in the averaging procedure.

This form of presentation makes the changes in horizontal anisotropy with changing environmental conditions more readily apparent. The trends match those 
TABLE 5. Summary of anisotropy results for large-eddy boundary layer cumulus simulations. Anisotropy directions are given using meteorological conventions.

\begin{tabular}{|c|c|c|c|c|c|c|}
\hline \multirow[b]{2}{*}{ Case } & \multicolumn{3}{|c|}{ Amplitude } & \multicolumn{3}{|c|}{ Direction } \\
\hline & $\begin{array}{c}\text { LWC } \\
\text { AP }\end{array}$ & Tilt & $\begin{array}{l}\text { LWP } \\
\text { AP }\end{array}$ & $\begin{array}{c}\text { LWC } \\
\text { AP }\end{array}$ & Tilt & $\begin{array}{c}\text { LWP } \\
\text { AP }\end{array}$ \\
\hline U0 & 0.011 & 0.011 & 0.024 & 193 & 225 & 259 \\
\hline U5 & 0.025 & 0.166 & 0.025 & 242 & 311 & 243 \\
\hline U10* & 0.034 & 0.473 & 0.110 & 313 & 308 & 307 \\
\hline U15 & 0.063 & 0.504 & 0.150 & 334 & 311 & 327 \\
\hline U20 & 0.077 & 0.731 & 0.190 & 336 & 311 & 325 \\
\hline SU5 & 0.080 & 0.266 & 0.098 & 270 & 268 & 277 \\
\hline SU10 & 0.229 & 0.649 & 0.384 & 273 & 273 & 273 \\
\hline SV5 & 0.084 & 0.336 & 0.152 & 259 & 267 & 256 \\
\hline SV10 & 0.049 & 0.509 & 0.142 & 214 & 229 & 225 \\
\hline F50 & 0.083 & 0.638 & 0.160 & 347 & 324 & 335 \\
\hline F150 & 0.029 & 0.314 & 0.082 & 285 & 305 & 293 \\
\hline BLW090 & 0.055 & 0.447 & 0.117 & 183 & 324 & 348 \\
\hline BLW110 & 0.019 & 0.221 & 0.067 & 297 & 312 & 311 \\
\hline
\end{tabular}

* This is the base case.

discussed above in terms of the spatial frequency bands. Overall horizontal anisotropy increases monotonically with both wind and shear amplitude when these factors are imposed independently. Addition of a shear orthogonal to a moderate mean wind yields somewhat stronger anisotropy than that associated with the mean wind alone. Increased forcing inhibits the development of spatial orientation in the cumulus fields, while anisotropy arises more readily when there is little forcing. Of the environmental factors examined, vertical speed shear parallel to the direction of the mean wind seems to produce the greatest horizontal anisotropy in the simulated fields.

\section{3) TILT}

The trends in the tilt results, also given in Table 5, are similar to those found for horizontal anisotropy except that the tilt is significant (greater than $0.031 \mathrm{~m} \mathrm{~m}^{-1}$ ) for every case with a nonzero initial wind. Tilt increases as the steady initial wind is increased, to a maximum of 0.731 when a $20 \mathrm{~m} \mathrm{~s}^{-1}$ wind is applied (U20). Tilt likewise increases with vertical speed shear. The $2 \mathrm{~m} \mathrm{~s}^{-1}$ $\mathrm{km}^{-1}$ zonal wind shear (SU5) produces more tilt than is computed for $5 \mathrm{~m} \mathrm{~s}^{-1}$ wind speeds (U5), and the tilt found in the case of the $4 \mathrm{~m} \mathrm{~s}^{-1} \mathrm{~km}^{-1}$ zonal wind shear (SU10) is between those computed for 15 and $20 \mathrm{~m} \mathrm{~s}^{-1}$ uniform initial wind speeds (U15 and U20). Significant tilt was also produced when meridional speed shear was imposed at the start of the simulation in addition to the standard uniform zonal wind (SV5 and SV10). However, while the tilt computed for case SV5 is greater than that for SU5, the tilt found for SV10 is much less than the tilt for SU10. The relatively low tilt detected for SV10 may be related to the larger change in wind direction with height that occurs in this case. For the cases in which the thermal forcing was altered, either by varying the surfaces fluxes (F50 and F150) or the water vapor profile (BLW090 and BLW110), greater tilt was produced when the convective forcing was weaker and less tilt developed when convection was stronger. Unlike the horizontal anisotropy, the greatest tilts occur for the cases with large initial uniform winds rather than those with the greatest imposed shear.

\section{4) Mechanisms}

From the preceding results, it is clear that both tilt and horizontal anisotropy increase with increasing wind speed and vertical shear. Still, it is not obvious which of these factors is more important to the development of anisotropy, since, even when uniform initial wind speeds are prescribed, shear develops as a result of friction at the surface. For example, the average cloudlevel shear in the ten scenes analyzed for the base case (with an imposed constant wind of $10 \mathrm{~m} \mathrm{~s}^{-1}$ ) is comparable to the shear in SU5. Likewise the average shear for U20 (with an imposed constant wind of $20 \mathrm{~m} \mathrm{~s}^{-1}$ ) is greater than the average shear in the high shear case SU10. To assess the relative importance of these factors, horizontal anisotropy and tilt for the LES liquid water content fields were correlated with in-cloud wind speed and shear. For ease of analysis, the anisotropy parameter values averaged over all spatial frequency bands were employed. Wind and shear information was taken from the vertical profile data output during the simulations. Only data for those scenes used in the anisotropy analysis were selected. Because the profile data were saved half as frequently as the threedimensional field data, wind data from just five scenes were used, on average. Wind and shear vectors were averaged over height within the cloudy levels of each selected scene, and then over all such scenes from each simulation. The average magnitudes and directions of anisotropy and tilt for all of the LES runs were then correlated with the amplitudes and directions of their mean winds and shears. Results are depicted in Figs. 9 and 10.

As shown in Fig. 9a, none of the LWC horizontal anisotropy can be attributed to wind speed alone. However, wind shear does account for a significant portion of the anisotropy, as evinced by the coefficient of determination of 0.463 given for this correlation in Fig 9b. On the other hand, some correlation between tilt and wind speed is evident. Nevertheless, far more of the variability in tilt $(90 \%)$ can be accounted for by the wind shear than by wind speed alone (48\%).

The correlations of directions in Fig. 10 further clarify these trends. Despite the moderate correlation between wind speed and tilt evident in Fig. 9, mean wind direction does not correlate well with the direction of either tilt or horizontal anisotropy. $\left(R^{2}<0.05\right.$ in both cases.) The average shear direction, on the other 

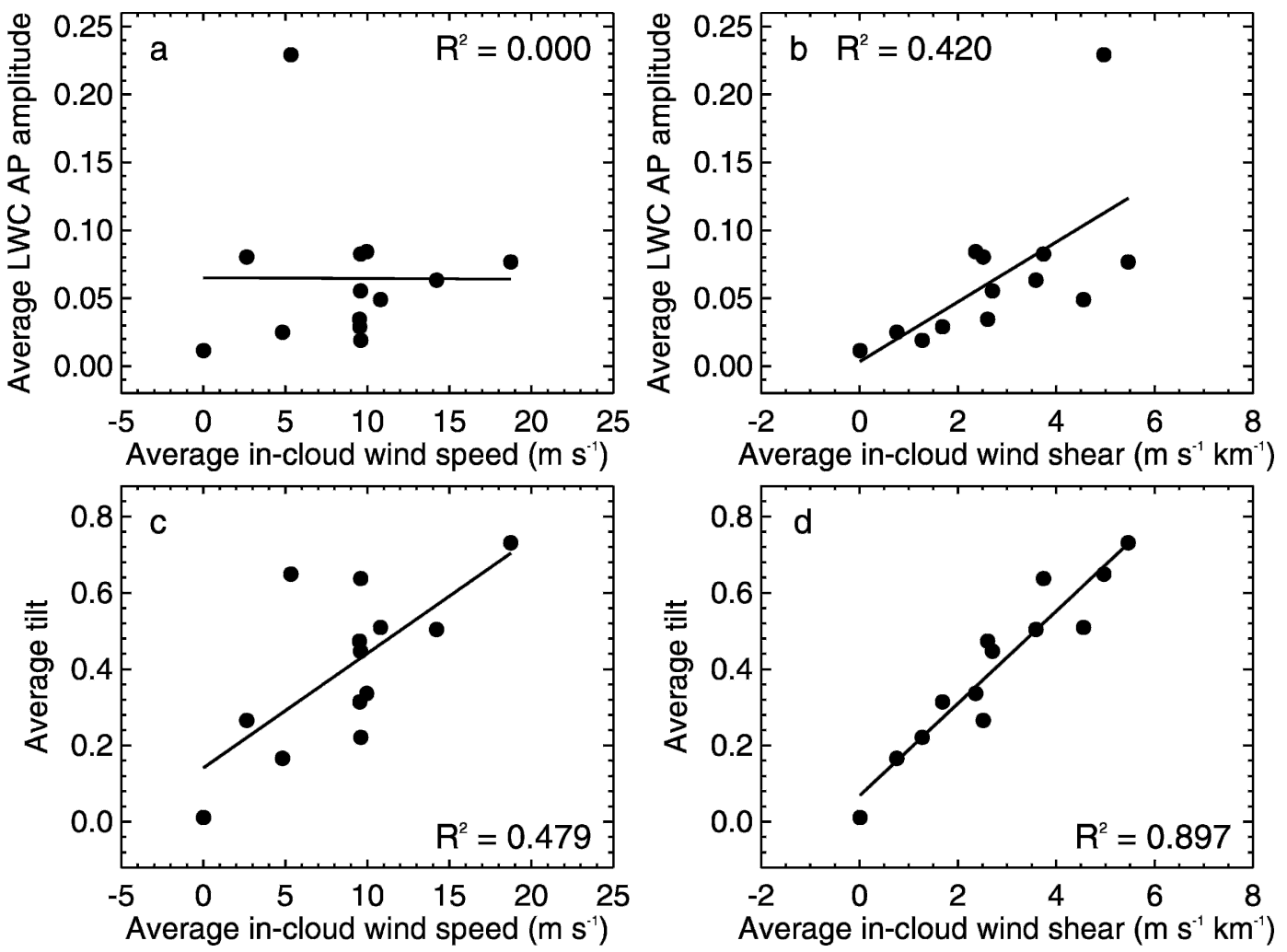

FIG. 9. Correlation of average anisotropy values with wind speed and shear. Best-fit lines are indicated.

hand, exhibits modest correlation with the direction of horizontal anisotropy and accounts for nearly all of the variability in tilt direction.

Together, these two analyses indicate that shear is much more important for the development of preferred directions in fair-weather cumulus cloud fields than strong winds alone. Although it is reasonable to expect that the wind speed and shear are themselves correlated in this study, their effects can be distinguished by their different orientations. Comparison of the average winds and shears in these LES cloud scenes, shown in Fig. 11, reveals that, while the amplitude of the wind speed and shear are somewhat correlated $\left(R^{2}=0.388\right)$, their mean directions are essentially uncorrelated $\left(R^{2}\right.$ $=0.053$ ), though the range of wind directions is admittedly small for this type of analysis. Thus the modest correlation between wind speed and tilt magnitudes evident in Fig. 9c may be due to the relationship between wind speed and shear amplitudes since shear rather than wind speed correlates well with tilt.

A similar analysis was carried out in terms of a nondimensional parameter relating buoyancy and shear (the ratio of mean shear to the cloud core updraft velocity over cloud depth). Presentation of this analysis has been omitted because the parameter was found to correlate more poorly with horizontal anisotropy and tilt than shear alone.

\section{Discussion}

As shown above, natural environmental phenomena, most significantly speed shear but also steady winds and weak surface forcing, give rise to tilt and horizontal anisotropy in fair-weather cumulus clouds. What the large-eddy simulations do not indicate, however, is the proportion of naturally occurring cumulus clouds that experience this stretching or tilting and the magnitude of the resulting distortion. These facts can be ascertained only through an extensive survey of cumulus cloud fields, which would likely require the use of satellite data.

Typical nadir-viewing satellites provide two-dimensional representations of cloud fields such as optical depth or radiance fields. Although the anisotropy parameter can be computed for such two-dimensional fields, without information about the vertical dimension the ability to distinguish between tilt and horizontal elongation is lost. For example, both a tilted cloud structure and a horizontally stretched element will appear elliptical once the field is projected into two dimensions. This limits the value of nadir-viewing satellites for the measurement of cloud anisotropy.

To illustrate this situation, the average horizontal anisotropy of the vertically integrated liquid water content, or liquid water path (LWP), fields from each 

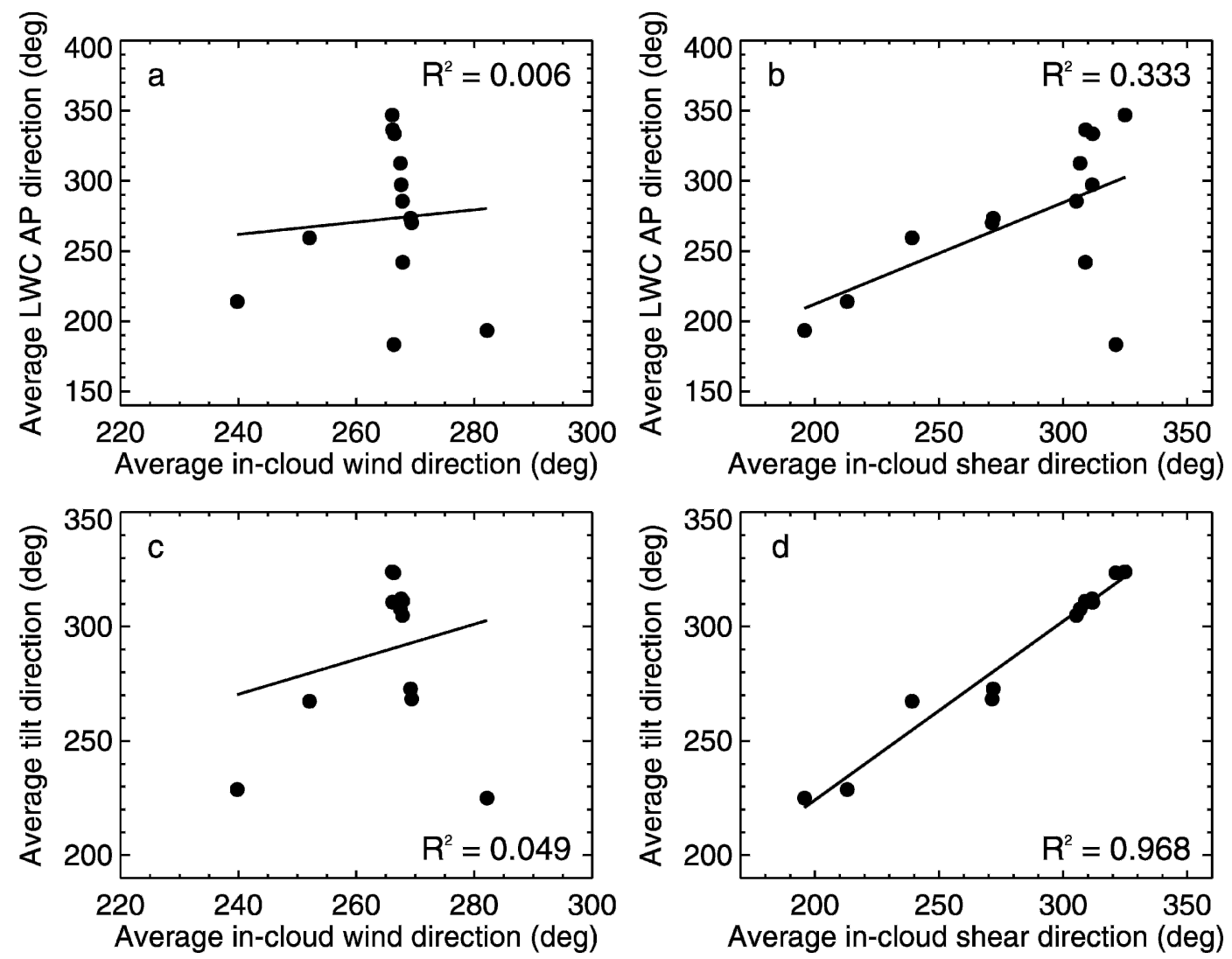

FIG. 10. Correlation of average anisotropy direction with average wind and shear directions.

large-eddy simulation is presented in Table 5 . The fact that the LWP anisotropy values exhibit trends similar to those observed for LWC anisotropy and tilt suggests that it may be possible to estimate the LWC parameters from the corresponding two-dimensional LWP anisotropy values. In fact, linear regression of the LWC anisotropy and tilt magnitudes against the LWP anisotropy values reveals moderate relationships between these pairs of variables. Using the resulting equations, LWC anisotropy and tilt can be computed to within \pm 0.017 and \pm 0.13 from LWP anisotropy, respectively. Since the specific cases simulated here were selected to isolate a few of the processes that influence cloud formation, they cannot be presumed representative of all cumulus clouds. Nevertheless, further investigation of the relationship between two- and three-dimensional anisotropy may prove useful.

Another approach to detecting cloud field anisotropy from space would be to employ satellite instruments with additional imaging capabilities. Multiangle satellite imagers, such as the Multiangle Imaging Spectroradiometer (MISR) and Advanced Spaceborne Thermal Emission and Reflection Radiometer (ASTER) on the satellite Terra, can provide some information about three-dimensional cloud structure, but have other disadvantages. MISR has several viewing angles but a resolution $(275 \mathrm{~m})$ that is several times coarser than the optimum for this study. ASTER has just two nearinfrared radiometers with views separated by $27.6^{\circ}$ but excellent resolution $(15 \mathrm{~m})$. Terra's orbit is sun-synchronous with a 1030 equator crossing so that most regions are not observed at times when cumulus clouds are most common over land (early afternoon in the summer hemisphere). Still, Terra should yield good sampling of marine cumuli. Despite the nonidealities of currently available instrumentation, a satellite survey could provide an indication of the severity and frequency of occurrence of cumulus cloud field anisotropy on a global scale.

Analyzing LES output in terms of anisotropy proves to be a useful diagnostic tool for dynamical models. For example, in section $4 \mathrm{~b}(1)$, it was observed that the horizontal anisotropy detected in the liquid water content fields was often most pronounced at the smaller spatial scales. In fact, the upturn at small scales was far more extreme for the original high shear runs done in this study. Because turbulence theory holds that flows should be more isotropic at smaller scales, these results were investigated in greater detail. It was found that a Galilean transformation had been applied incorrectly in these cases. In the UCLA LES model, a uniform base wind field is subtracted from the initial profile and only 

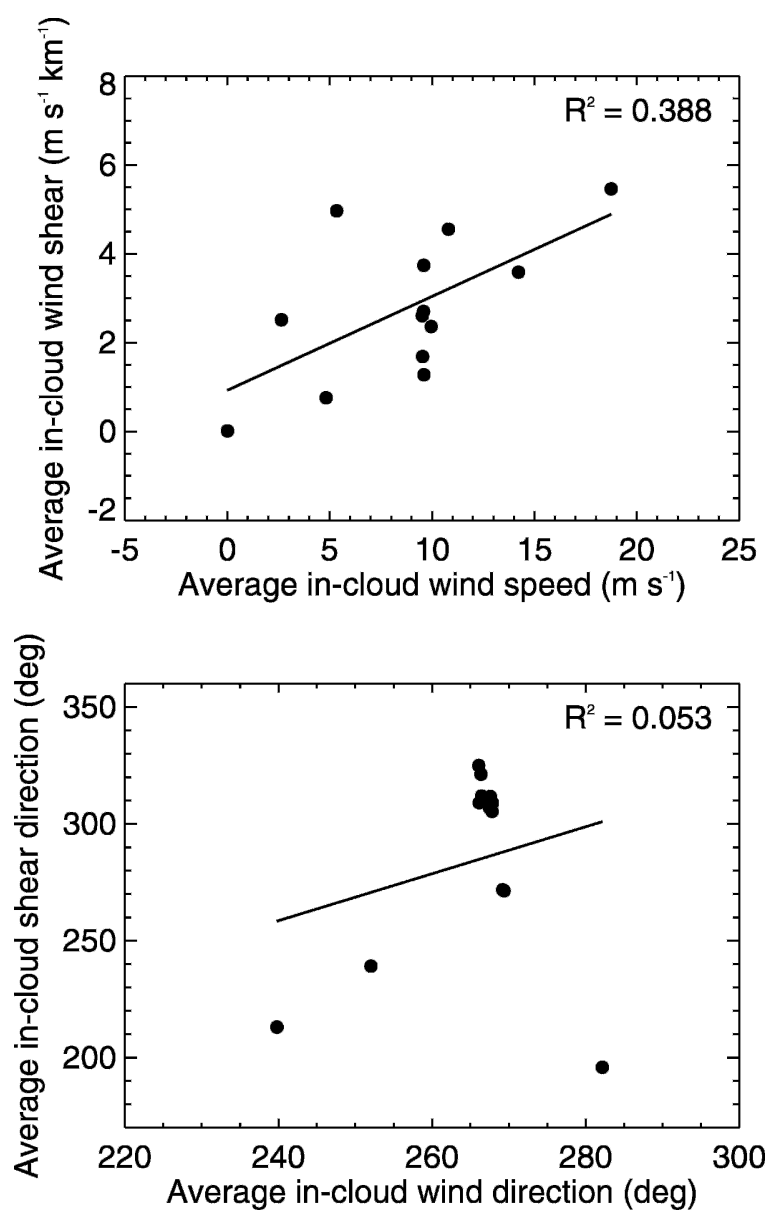

FIG. 11. Correlation of shear and wind speeds and directions in large-eddy simulations.

the deviations from this field are carried through most of the calculations. (The total velocities are used where necessary, such as in computing surface friction.) This allows a larger time step to be used for the same Courant-Friedrich-Levy (CFL) criterion. For best performance, the base wind specified should be close to the mean wind throughout the simulation, but for the shear cases it had been chosen incorrectly. Absent the anisotropy analysis, this error might have gone undetected because the profiles and spectra of other dynamical and thermodynamical fields typically examined in model studies did not appear unusual. Though the error was corrected and only the later results are presented in this paper, our diagnostics do suggest that the fidelity with which the model is able to represent high shear flow in the presence of cumulus convection at scales near the grid scale may be limited.

The reader is cautioned not to interpret the results of this study too broadly since they are based entirely on continental fair-weather cumulus-type fields created by a single LES model. Because of the computational demands, only a few different environmental forcings were considered and combinations of forcings were not investigated. Simplifications in the LES and base scenario meant that the effects of cloud microphysics and radiative processes based on the simulated cloud structures were omitted. In addition, only the base case was rerun with different random potential temperature variations to test the natural variability of the results. Despite these constraints, we expect that the correlations between environmental factors and the development of tilt or anisotropy observed here for fairweather cumulus clouds are robust, particularly the dominant role of shear.

The way in which the LES generates horizontal anisotropy and tilt in response to environmental conditions might best be thought of as a nontrivial prediction of the model, testable with observations. Given the limitations of satellites in this respect, a combination of airborne and surface-based remote sensing instruments might provide the best opportunity to evaluate the degree to which nature conforms to specific LES predictions. The Rain in Cumulus over the Ocean (RICO) field experiment, scheduled to take place from November 2004 through January 2005, will provides an opportunity for such tests.

\section{Conclusions}

The purpose of this research was to evaluate the extent to which various meteorological conditions give rise to anisotropy in fair-weather cumulus cloud fields on scales of tens to hundreds of meters. This question was investigated by simulating the formation of cumulus clouds under different environmental conditions using a large-eddy simulation (LES) model. The model initial and boundary conditions were selected to simulate the development of shallow cumulus over the southern Great Plains due to convection driven by surface forcing. The mean initial wind speed, wind shear, water vapor mixing ratio, and time-dependent surface fluxes were varied in turn and the horizontal anisotropy and tilt of the clouds in output scenes from these simulations were computed using newly developed quantitative measures. Vertical speed shear was found to be most highly correlated with the development of both vertically tilted and horizontally stretched cloud structures. High mean wind speed was also associated with vertical tilt, but this may be due in part to the higher wind shears that accompany strong mean winds. Additional convective forcing, whether in the form of increased surface fluxes or latent heat release, appeared to inhibit cloud organization. The greatest anisotropy amplitudes were observed for the smallest spatial scales. It was noted that cloud field tilt and stretching cannot be distinguished when cloud fields are represented as two-dimensional projections, implying that horizontal anisotropy and tilt cannot be uniquely identified in most satellite images. Alternative approaches 
to measuring cloud anisotropy on a large scale were suggested.

Acknowledgments. The authors greatly appreciate the extensive advice on turbulence theory, cloud formation, large-eddy simulation, and scale interactions provided by John Wyngaard, Jerry Harrington, and Natasha Miles of The Pennsylvania State University. Eugene Clothiaux is thanked for his guidance and for supplying the idealized fields used to illustrate the anisotropy parameter. LMH is grateful to Thomas Ackerman for his advice and financial support. This material is based upon work supported under a National Science Foundation Graduate Fellowship. Additional funding was provided by the Department of Energy through Grants DE-FG02-90ER-61071 and DE-A1005-90ER61069, and Battelle, Inc., Contracts 354048-AQ5 and 354879-AQ5, and by the National Science Foundation through Grant ATM-9985413.

\section{REFERENCES}

Aida, M., 1977: Reflection of solar radiation from an array of cumuli. J. Meteor. Soc. Japan, 55, 174-181.

Barker, H. W., 1994: Solar radiative transfer for wind-sheared cumulus cloud fields. J. Atmos. Sci., 51, 1141-1156.

— scale-invariant broken cloud fields. J. Atmos. Sci., 49, 11151126.

Bretherton, C. S., and Coauthors, 1999: An intercomparison of radiatively driven entrainment and turbulence in a smoke cloud, as simulated by different numerical models. Quart. J. Roy. Meteor. Soc., 125, 391-423.

Brown, A. R., 1999: Large-eddy simulation and parametrization of the effects of shear on shallow cumulus convection. Bound.-Layer Meteor., 91, 65-80.

—_, and Coauthors, 2002: Large-eddy simulation of the diurnal cycle of shallow cumulus convection over land. Quart. J. Roy. Meteor. Soc., 128, 1075-1093.

Busygin, V. P., N. A. Yevstratov, and Y. M. Feygel'son, 1973: Optical properties of cumulus clouds, and radiant fluxes for cumulus cloud cover. Izv. Acad. Sci. USSR, Atmos. Oceanic Phys., 9, 1142-1151.

Cahalan, R. F., W. Ridgway, W. J. Wiscombe, S. Gollmer, and Harshvardhan, 1994: Independent pixel and Monte Carlo estimates of stratocumulus albedo. J. Atmos. Sci., 51, 37763790.

Cho, H.-R., 1978: Some statistical properties of a homogeneous and stationary shallow cumulus cloud field. J. Atmos. Sci., 35, $125-138$.

Christian, T. W., 1987: A comparative study of the relationship between radar reflectivities, Doppler velocities, and clouds associated with horizontal convective rolls. M.S. thesis, Dept. of Atmospheric Sciences, University of California, Los Angeles, $94 \mathrm{pp}$.

Couteron, P., and O. Lejeune, 2001: Periodic spotted patterns in semi-arid vegetation explained by a propagation-inhibition model. J. Ecol., 89, 616-628.

Derbyshire, E., D. J. Unwin, X. M. Fang, and M. Langford, 1992: The Fourier frequency-domain representation of sediment fabric anisotropy. Comput. Geosci., 18, 63-73.

Etling, D., and R. A. Brown, 1993: Roll vortices in the planetary boundary layer: A review. Bound.-Layer Meteor., 65, 215248.

Evans, K. F., and W. J. Wiscombe, 2004: An algorithm for generating stochastic cloud fields from radar profile statistics. Atmos. Res., 72, 263-289.
Glendening, J. W., 2000: Budgets of lineal and nonlineal turbulent kinetic energy under strong shear conditions. J. Atmos. Sci., 57, 2297-2318.

Grossman, R. L., 1982: An analysis of vertical velocity spectra obtained in the BOMEX fair-weather, trade-wind boundary layer. Bound.-Layer Meteor., 23, 323-357.

Harshvardhan, and R. W. L. Thomas, 1984: Solar reflection from interacting and shadowing cloud elements. J. Geophys. Res., 89, 7179-7185.

Heymsfield, A. J., P. N. Johnson, and J. E. Dye, 1978: Observations of moist adiabatic ascent in northeast Colorado cumulus congestus clouds. J. Atmos. Sci., 35, 1689-1703.

Houghton, J. T., Y. Ding, D. J. Griggs, M. Noguer, P. J. van der Linden, X. Dai, K. Maskell, and C. A. Johnson, Eds., 2001: Climate Change 2001: The Scientific Basis. Cambridge University Press, $881 \mathrm{pp}$.

Joseph, J. H., 1985: The morphology of fair-weather cumulus clouds as remotely sensed from satellites and some applications. Adv. Space Res., 5, 213-216.

Kuettner, J. P., 1971: Cloud bands in the earth's atmosphere: Observations and theory. Tellus, 23, 404-425.

LeMone, M. A., 1973: The structure and dynamics of horizontal roll vortices in the planetary boundary layer. J. Atmos. Sci., 30, 1077-1091.

Marshak, A., A. Davis, W. Wiscombe, and R. Cahalan, 1995: Radiative smoothing in fractal clouds. J. Geophys. Res., 100, $26247-26261$

Mason, P. J., 1992: Large-eddy simulation of dispersion in convective boundary layers with wind shear. Atmos. Environ., 26A, 1561-1571.

McKee, T. B., and S. K. Cox, 1974: Scattering of visible radiation by finite clouds. J. Atmos. Sci., 31, 1885-1892.

Peng, Z., and T. B. Kirk, 1997: Two-dimensional fast Fourier transform and power spectrum for wear particle analysis. Tribol. Int., 30, 583-590.

Pincus, H. J., and M. B. Dobrin, 1966: Geological applications of optical data processing. J. Geophys. Res., 71, 4861-4869.

Press, W. H., S. A. Teukolsky, W. T. Vetterling, and B. P. Flannery, 1992: Numerical Recipes in C: The Art of Scientific Computing. 2d ed. Cambridge University Press, 994 pp.

Prince, C. M., and R. Ehrlich, 1990: Analysis of spatial order in sandstones. I. Basic principles. Math. Geol., 22, 333-359.

Renshaw, E., and E. D. Ford, 1984: The description of spatial pattern using two-dimensional spectral analysis. Vegetatio, 56, 75-85.

Saffman, P. G., 1962: The effect of wind shear on horizontal spread from an instantaneous ground source. Quart. J. Roy. Meteor. Soc., 88, 382-393.

Sengupta, S. K., R. M. Welch, M. S. Navar, T. A. Berendes, and D. W. Chen, 1990: Cumulus cloud field morphology and spatial patterns derived from high spatial resolution Landsat imagery. J. Appl. Meteor., 29, 1245-1267.

Shirer, H. N., 1986: On cloud street development in three dimensions: Parallel and Rayleigh instabilities. Contrib. Atmos. Phys., 49, 126-149.

Stevens, B., C.-H. Moeng, and P. P. Sullivan, 1999: Large-eddy simulation of radiatively driven convection: Sensitivities to the representation of small scales. J. Atmos. Sci., 56, 39633984.

- and Coauthors, 2001: Simulations of trade wind cumuli under a strong inversion. J. Atmos. Sci., 58, 1870-1891.

Sykes, R. I., and D. S. Henn, 1989: Large-eddy simulation of turbulent sheared convection. J. Atmos. Sci., 46, 1106-1118.

Taylor, A. D., 1982: Puff growth in an Ekman layer. J. Atmos. Sci., 39, $837-850$

Várnai, T., and R. Davies, 1999: Effects of cloud heterogeneities on shortwave radiation: Comparison of cloud-top variability and internal heterogeneity. J. Atmos. Sci., 56, 4206-4224.

Weckwerth, T. M., J. W. Wilson, R. M. Wakimoto, and N. A. Crook, 1997: Horizontal convective rolls: Determining the 
environmental conditions supporting their existence and characteristics. Mon. Wea. Rev., 125, 505-526.

Welch, R. M., and B. A. Wielicki, 1984: Stratocumulus cloud field reflected fluxes: The effect of cloud shape. J. Atmos. Sci., 41, 3085-3103.

Wood, R., and D. Hartmann, 2001: Investigations of LWP spatial variability using MODIS. Proc. 11th Conf. on Satellite Meteorology and Oceanography, Madison, WI, Amer. Meteor. Soc., $168-171$.
Zhang, M. H., J. L. Lin, R. T. Cederwall, J. J. Yio, and S. C. Xie, 2001: Objective analysis of ARM IOP data: Method and sensitivity. Mon. Wea. Rev., 129, 295-311.

Zhu, T., J. Lee, R. C. Weger, and R. M. Welch, 1992: Clustering, randomness and regularity in cloud fields: 2 . Cumulus cloud fields. J. Geophys. Res., 97, 20 537-20 558.

Zuidema, P., and K. F. Evans, 1998: On the validity of the independent pixel approximation for boundary layer clouds observed during ASTEX. J. Geophys. Res., 103, 6059-6074. 